\title{
Cross-colonisation with Pseudomonas aeruginosa of patients in an intensive care unit
}

Dennis C J J Bergmans, Marc J M Bonten, Frank H van Tiel, Carlo A Gaillard, Siebe van der Geest, Rob M Wilting, Peter W de Leeuw, Ellen E Stobberingh

\begin{abstract}
Background-Ventilator-associated pneumonia (VAP) caused by Pseudomonas aeruginosa is usually preceded by colonisation of the respiratory tract. During outbreaks, colonisation with $P$ aeruginosa is mainly derived from exogenous sources. The relative importance of different pathways of colonisation of $P$ aeruginosa has rarely been determined in non-epidemic settings.
\end{abstract}

Methods-In order to determine the importance of exogenous colonisation, all isolates of $P$ aeruginosa obtained by surveillance and clinical cultures from two identical intensive care units (ICUs) were genotyped with pulsed field gel electrophoresis.

Department of Internal Medicine, University Hospital Maastricht, Maastricht, The Netherlands

D C J J Bergmans

$S$ van der Geest

R M Wilting

$\mathrm{P} W$ de Leeuw

Department of Internal Medicine, University Hospital

Utrecht, Utrecht, The

Netherlands

M J M Bonten

Department of Medical Microbiology,

University Hospital

Maastricht,

Maastricht, The

Netherlands

$\mathrm{F} H$ van Tiel

E E Stobberingh

Department of Internal Medicine, Eemland Hospital, Amersfoort, The

Netherlands

C A Gaillard

Correspondence to: Dr D C J J Bergmans, Department of Internal Medicine, University

Hospital Maastricht, P.O.

Box 5800, 6202 AZ

Maastricht, The

Netherlands.

Received 9 February 1998 Returned to author Returned to a
18 May 1998

Revised manuscript received

17 June 1998

Accepted for publication

6 July 1998
Results-A total of 100 patients were studied, 44 in ICU 1 and 56 in ICU 2. Twenty three patients were colonised with $P$ aeruginosa, seven at the start of the study or on admission and 16 of the remaining 93 patients became colonised during the study. Eight patients developed VAP due to $P$ aeruginosa. The incidence of respiratory tract colonisation and VAP with $P$ aeruginosa in our ICU was similar to that before and after the study period, and therefore represents an endemic situation. Genotyping of 118 isolates yielded 11 strain types: eight in one patient each, two in three patients each, and one type in eight patients. Based on chronological evaluation and genotypical identity of isolates, eight cases of cross-colonisation were identified. Eight (50\%) of 16 episodes of acquired colonisation and two (25\%) of eight cases of VAP due to $P$ aeruginosa seemed to be the result of crosscolonisation.

Conclusions-Even in non-epidemic settings cross-colonisation seems to play an important part in the epidemiology of colonisation and infection with $P$ aeruginosa.

(Thorax 1998;53:1053-1058)

Keywords: colonisation; Pseudomonas aeruginosa; ventilator-associated pneumonia

Ventilator-associated pneumonia (VAP) is the most important nosocomial infection among mechanically ventilated patients in intensive care units (ICUs) and is associated with increased morbidity and mortality, especially when caused by Pseudomonas aeruginosa. ${ }^{1}$ VAP is almost always preceded by colonisation of the upper respiratory tract with the causative microorganism. ${ }^{2}$ However, the exact routes of colonisation leading to VAP and the relative importance of different pathways of colonisation is a matter of controversy. ${ }^{34}$

Respiratory tract colonisation with $P$ aeruginosa may originate from endogenous sources such as the intestine or the stomach, ${ }^{5-7}$ or from exogenous sources such as contaminated equipment or other patients colonised with $P$ aeruginosa..$^{8-10}$ Understanding the relative importance of these routes of colonisation is crucial to the development of effective preventive measures against infection. If, for example, cross-colonisation occurs frequently, improved compliance with infection control measures will be necessary, but such a measure will be less effective if most infections are from endogenous sources. In the latter case, modulation of bacterial colonisation of the gastrointestinal tract may be more appropriate. The clinical observation of three episodes of VAP due to $P$ aeruginosa in a short period of time was the starting point for the present study. Infection was assumed to be the result of crosscolonisation of microorganisms. In order to determine the importance of exogenous and endogenous colonisation of $P$ aeruginosa we initiated an analysis of colonisation and infection with this pathogen.

\section{Methods}

The study was performed in two identical eight bed intensive care units (ICU 1 and ICU 2) of the University Hospital Maastricht, a 700 bed hospital. Both ICUs contained a mixed population of surgical, medical, neurosurgical, and trauma patients who were randomly admitted. The units have different nursing staff and medical equipment, but the medical staff and paramedic care providers (physiotherapists, radiographic personnel, and the nutrition support team) have patient contacts in both ICUs. No outbreaks of multiple resistant microorganisms have occurred during the last five years.

PATIENTS

All patients admitted to ICU 1 and ICU 2 (ventilated or non-ventilated) were studied prospectively with regard to respiratory tract colonisation and the development of VAP and other ICU acquired infections in two study periods: from 6 November 1993 to 15 December 1993 in ICU 1 (40 days) and from 17 November 1993 to 15 January 1994 in ICU 2 (60 days). The following data were recorded on admission: age, sex, medical specialty, length of time in hospital before admission to the ICU, 
diagnosis on admission, reason for intubation, previous medical history, and APACHE II score. ${ }^{11}$ During the stay in the ICU clinical parameters (temperature, leucocyte count, chest radiographs) were recorded daily. All 16 mechanically ventilated patients receiving enteral nutrition with an expected duration of ventilation of $\geqslant 3$ days were included in a prospective study on the influence of continuous and intermittent enteral feeding on respiratory and digestive tract colonisation. ${ }^{12}$ Another six patients were included in a study on sequences of colonisation within the patient.

SURVEILLANCE CULTURES

Patients were subjected to microbiological examination of endotracheal aspirates and urine on admission and then twice weekly. In addition, a more extensive examination was performed on the patients included in the two prospective studies mentioned above. Gastric aspirates and oropharyngeal swabs were cultured daily from the 16 patients in the study on the effects of intermittent enteral feeding, and from the six patients studied to determine sequences of colonisation cultures were obtained twice daily (08.00 and 16.00 hours) from six body sites (nose, oropharynx, sputum, gastric aspirate, rectum, and groin) during their stay in the ICU. The results of all clinical cultures were also included in the analysis. No cultures were taken from non-intubated patients with an ICU stay of less than 24 hours.

DEFINITIONS

(1) Colonisation was defined as the isolation of microorganisms from specimens taken from any body site in the absence of infection. Colonisation on admission was defined as that occurring within 48 hours of admission to the ICU. When colonisation occurred more than 48 hours after admission to the ICU, without colonisation on admission, it was defined as acquired colonisation.

(2) Ventilator-associated pneumonia was diagnosed by bronchoscopy with protected specimen brush (PSB) and bronchoalveolar lavage (BAL). The diagnosis was established when (a) at least three of the following criteria were met: (i) rectal temperature above $38.0^{\circ} \mathrm{C}$ or below $35.5^{\circ} \mathrm{C}$, (ii) blood leucocytosis $(>10 \times$ $\left.10^{3} / \mathrm{mm}^{3}\right)$ and/or left shift or blood leucopenia $\left(<3 \times 10^{3} / \mathrm{mm}^{3}\right)$, (iii) more than 10 leucocytes per high power field in gram stain of tracheal aspirate, and (iv) a positive culture from a tracheal aspirate, in combination with (b) a new or progressive infiltrate on the chest radiograph, and (c) the presence of (i) a positive quantitative culture of a sample of secretions obtained by BAL (cutoff point $\geqslant 10^{4} \mathrm{cfu} / \mathrm{ml}$ ) or PSB (cutoff point $\geqslant 10^{3} \mathrm{cfu} / \mathrm{ml}$ ) or (ii) positive cultures from blood unrelated to another source obtained within 48 hours before and after respiratory sampling and with the same species as cultured from the tracheal aspirate, or (iii) positive pleural fluid culture in the absence of previous pleural instrumentation. Pneumonia was considered to be ICUacquired if symptoms began $\geqslant 48$ hours after admission to the ICU.
(3) Endogenous colonisation was defined as colonisation occurring with a strain of $P$ aeruginosa that had not previously been isolated from another patient.

(4) Exogenous colonisation or crosscolonisation was defined as colonisation occurring with a strain of $P$ aeruginosa with similar pulsed field gel electrophoresis (PFGE) typing results to that of isolates from another patient present in one of the ICUs.

\section{COLONISATION PRESSURE AND ANTIBIOTIC}

PRESSURE

For each study day the point prevalence of $P$ aeruginosa colonisation in the ICU was calculated as follows: number of patients colonised with $P$ aeruginosa on that day divided by the total number of patients present in the ICU on that day. Subsequently, for each patient who was not colonised with $P$ aeruginosa on admission or at the start of the study, the mean point prevalence of $P$ aeruginosa colonisation for all days in the ICU until acquisition of $P$ aeruginosa or until discharge (if the patient did not acquire colonisation) was calculated. This calculated number reflects the colonisation pressure with $P$ aeruginosa for the period in the ICU that the patient was not colonised.

Antibiotic pressure was determined as follows: the percentage of days that non-colonised patients received antibiotic therapy that provided $P$ aeruginosa with a selective growth advantage ((amino)penicillins, first and second generation cephalosporins, macrolides, glycopeptides etc) was calculated until acquisition of $P$ aeruginosa or until discharge from the ICU.

MICROBIOLOGY AND BACTERIAL TYPING

Semi-quantitative microbiological analysis of surveillance and clinical cultures were performed and PSB and BAL samples were analysed quantitatively. All isolated species were identified according to methods routinely used in the medical microbiology laboratory. Specimens were inoculated immediately after sampling, seven days a week. From each culture plate with growth of $P$ aeruginosa three colonies were analysed and stored separately until further processing. All isolates of $P$ aeruginosa were stored at a temperature of $-70^{\circ} \mathrm{C}$. From each patient and each body site found to be colonised with $P$ aeruginosa isolates were randomly selected for bacterial typing. The antibiotic susceptibility pattern was determined by measurement of minimal inhibitory concentrations using the micro-broth dilution method according to the NCCLS guidelines. $P$ aeruginosa ATCC 27853 and Escherichia coli ATCC 25922 and 35218 were used as reference strains. Susceptibility to the following antibiotics was determined: piperacillin, coticarclavulanate, ceftazidime, imipenem, gentamicin, tobramycin, amikacin, ciprofloxacin, and ofloxacin. PFGE was carried out according to the method described by Grothues et $a l^{13}$ using a CHEF-DRII System (Bio-Rad Laboratories, Richmond, California, USA) after restriction digestion with SpeI (Boehringer Mannheim, Germany). Saccharomyces cerevisiae was used as a marker. 
Table 1 Patient characteristics

\begin{tabular}{llll}
\hline & ICU 1 & ICU 2 & Total \\
\hline Number of patients studied & 44 & 56 & 100 \\
Number of colonised patients & 9 & 14 & 23 \\
$\quad$ Prior to study period & 1 & 2 & 3 \\
On admission & 4 & 0 & 4 \\
$\quad$ Acquired & 4 & 12 & 16 \\
Number of pneumonias & 3 & 5 & 8 \\
Median age (range) & $61(0-92)$ & $61(0-79)$ & $61(0-92)$ \\
Male/female & $25 / 19$ & $30 / 26$ & $55 / 45$ \\
Median APACHE II score (range) & $21(5-33)$ & $18(2-41)$ & $19(2-41)$ \\
Median days in ICU (range) & $3(1-535)$ & $4(1-68)$ & $4(1-535)$ \\
Hospital stay prior to ICU^ & $2(0-147)$ & $2(0-115)$ & $2(0-147)$ \\
Medical specialty & 12 & 12 & 24 \\
$\quad$ Surgery/trauma & 10 & 17 & 27 \\
Medical & 5 & 6 & 11 \\
Pulmonology & 7 & 10 & 17 \\
Neurology/neurosurgery & 4 & 1 & 5 \\
Paediatrics & 3 & 4 & 7 \\
Cardiology & 3 & $14(25 \%)$ & $27(27 \%)$ \\
$\quad$ Other & $13(30 \%)$ & & \\
Mortality & & & \\
\hline
\end{tabular}

^ Median (range) days in hospital before admission to ICU.

PFGE patterns were compared visually on enlarged gel photographs to identify bands of identical relative mobility as fragments of identical size. The absolute number of band differences between pairs of isolates was recorded. The criteria of Tenover et $a l^{14}$ were used to interpret chromosomal DNA restriction patterns produced by PFGE for typing of bacterial strains. Indistinguishable isolates (no band differences) and closely related isolates ( $2-3$ band differences) were considered to be the same genotype, while possibly related and different isolates ( $4-6$ and $\geqslant 7$ band differences, respectively) were considered different genotypes.

STATISTICAL ANALYSIS

Data are expressed as absolute numbers with or without percentages, as means with standard deviation (SD), or as medians with ranges. Frequency comparisons were performed by the Mann-Whitney test or $\chi^{2}$ test, a $p$ value of less than 0.05 being considered statistically significant.

\section{Results}

In all, 100 patients were studied of whom 65 were mechanically ventilated. During the study period in ICU 1 (40 days) 44 patients were studied-40 were admitted and four were present at the start of the study. In ICU 2 (60 days) 56 patients were studied, 50 of whom were admitted and six were present at the onset of the study. The characteristics of the patients admitted to both ICUs were comparable (table 1). Furthermore, the occupancy rate of beds was similar in both ICUs, $91 \%$ in ICU 1 and $93 \%$ in ICU 2 .

SURVEILLANCE OF COLONISATION

In all, 1370 specimens were taken from 73 patients (243 endotracheal aspirates, 159 urine samples, $383 \mathrm{blood} /$ pus/intravascular lines, 47 urinary catheters, $28 \mathrm{PSB} / \mathrm{BAL}$ fluid samples, 199 oropharyngeal swabs, 40 rectal swabs, 191 gastric aspirates, 41 nose swabs, and 39 groin swabs). No cultures were taken from 27 patients because their stay in the ICU was less than 24 hours and they were not intubated. Most of these patients were postoperative neurosurgical patients and patients admitted after auto-intoxication. $P$ aeruginosa was isolated from 69 specimens (22 endotracheal aspirates, two urine samples, 15 blood/pus/ intravascular lines, three urinary catheters, seven PSB/BAL fluid samples, 11 oropharyngeal swabs, two rectal swabs, five gastric aspirates, one nose swab and one groin swab) obtained from 23 patients. Eight of them developed VAP. Surveillance cultures were positive for $P$ aeruginosa before this species was isolated from clinical cultures in $77 \%$ of cases.

Colonised patients had a longer median stay in the ICU than non-colonised patients (25 versus 3 days, $\mathrm{p}<0.0001)$, were more frequently intubated $(96 \%$ versus $62 \%, \mathrm{p}<0.01$ ), and had a longer median duration on mechanical ventilation ( 17 versus 2 days, $\mathrm{p}<0.0001$ ), but did not differ significantly with respect to the other characteristics (data not shown).

Four $(4.4 \%)$ of the 90 patients admitted to the ICUs were colonised with $P$ aeruginosa on admission, all of them in ICU 1 . In addition, three out of 10 patients who were present in the ICU at the start of the study were already colonised, one in ICU 1 and two in ICU 2. Thus, 93 patients were not colonised with $P$ aeruginosa at the onset of the study or on admission to the ICU, and $16(17 \%)$ of them acquired colonisation with $P$ aeruginosa, four of 39 in ICU 1 and 12 of 54 in ICU 2.

\section{VENTILATOR-ASSOCIATED PNEUMONIA}

During the study period eight (8\%) of 100 patients developed VAP caused by $P$ aeruginosa (table 1), three in ICU 1 and five in ICU 2, with a mean (SD) duration from admission to ICU to diagnosis of VAP of 7.3 (3.1) days. The incidence of VAP caused by $P$ aeruginosa was $8 \%$ in the total study population. However, the incidence among ventilated patients was $12 \%$ (eight of 65 ) and $20 \%$ (eight of 41 ) for patients ventilated for $\geqslant 3$ days. The overall incidence of VAP caused by $P$ aeruginosa during the total study period was 0.9 per 100 patient days. In periods of two months the incidence of VAP caused by $P$ aeruginosa ranged from 0.4 to 0.9 per 100 patient days in the year following the study period, suggesting that the incidence of VAP during the period of study was not exceptional in our ICU.

MICROBIOLOGICAL TYPING

In all, 118 isolates of $P$ aeruginosa were genotyped, cultured from the following sites: 61 endotracheal aspirates, seven blood samples, two intravascular lines, $21 \mathrm{PSB} / \mathrm{BAL}$ fluid samples, 18 oropharynx, and nine stomach samples. In two patients (B and S) P aeruginosa was cultured only once during the stay in the ICU. Unfortunately these isolates were lost in the process of storage and could not be genotyped.

Antimicrobial susceptibility patterns were similar for isolates of all but one patient - that is, susceptible to piperacillin, coticarclavulanate, ceftazidime, imipenem, gentamicin, tobramycin, amikacin, ciprofloxacin and ofloxacin. One patient $(\mathrm{J})$ was colonised with a strain of $P$ aeruginosa resistant to 
gentamicin and tobramycin (type X), and such a strain was not isolated from any other patient. We assumed therefore that cross-colonisation with this strain did not occur and isolates from this patient were not genotyped. Using PFGE, 11 different genotypes could be distinguished (types 1-11). Eight genotypes were found in single patients only, whereas one genotype (type 2) was isolated from eight patients and two genotypes (type 3 and type 7) were isolated from three patients. Two patients (U and W) were colonised with two different genotypes each.

INCIDENCE OF CROSS-COLONISATION

Genotype 2 was isolated from eight patients, five in ICU 1 and three in ICU 2 (fig 1). Patient $M$ (ICU 2) was colonised with genotype 2 at the start of the study. Patient T (ICU 1) acquired genotype 2, presumably via crosscolonisation from patient $M$. Subsequently, genotype 2 colonised patient $\mathrm{O}$ (ICU 1) and patient K (ICU 2). Since patients $M$ and K were not in ICU 2 simultaneously, patient $\mathrm{K}$ most probably acquired genotype 2 via patient $\mathrm{T}$ in ICU 1 . Acquisition of genotype 2 by patient $\mathrm{D}$ could have been the result of cross-colonisation with patients $\mathrm{U}$ or $\mathrm{R}$ from ICU 1 as donor, or with patient $\mathrm{K}$ in ICU 2 as donor. Patients K and D were in ICU 2 simultaneously, but cultures of patient D only became positive six days after discharge of patient $\mathrm{K}$. This delay may have been caused by sampling error or because colonisation initially occurred in numbers below the detection threshold of culture techniques. Three other patients in ICU 1 were colonised with genotype 2 when they were admitted (patients $Q, R$ and $\mathrm{U})$. Two of them were admitted directly from the community and one patient had been admitted to a general hospital ward one day before ICU admission. Cultures from all three patients were taken within 24 hours of admission. In patient $\mathrm{Q}$, admitted directly from the community, genotype 2 was cultured from the endotracheal aspirate on admission. This patient had been treated in the ICU four months earlier and was colonised with $P$ aeruginosa during that time as well. Unfortunately no strains from that period were genotyped. Thus, four patients ( $\mathrm{T}$ and $\mathrm{O}$ in ICU 1 , and $\mathrm{K}$ and D in ICU 2) appeared to have acquired genotype 2 by cross-colonisation. At the start of the study patient $\mathrm{H}$ (ICU 2) was colonised with genotype 3. Subsequently, colonisation with this genotype was demonstrated in patient A (on the first day of the study) and patient I (both in ICU 2). Although cultures from patient I only became positive with genotype 3 after discharge of patients $\mathrm{A}$ and $\mathrm{H}$, all three patients were treated simultaneously in ICU 2 for a number of days. Finally, patient C (ICU 2) acquired colonisation with genotype 7 , presumably from an endogenous source. Subsequently, two other patients in ICU $2(\mathrm{~F}$ and $\mathrm{N})$ acquired colonisation with this genotype. All other patients acquired colonisation with a unique genotype which was therefore presumed to be derived from endogenous sources. In all, eight episodes of cross-colonisation were
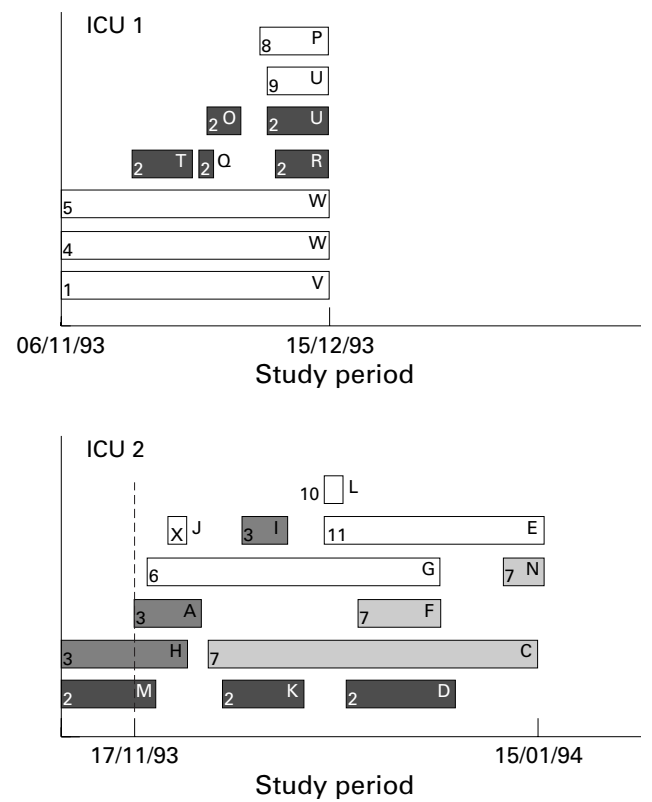

Genotype $2 \square$ Genotype 3

Genotype $7 \square$ Other

Figure 1 Colonisation of $P$ aeruginosa genotypes. Each horizontal bar represents the length of time a patient was colonised during the study period. Patient characters (in capitals) and genotypes (in numbers) are depicted within or just adjacent to the bars. Genotypes colonising more than one patient (types 2, 3 and 7) have marked bars. Blank bars represent the genotypes that were found in single patients only (types 1, 4, 5, 6, 8, 9, 10 and 11). Type $X$ represents the strain resistant to gentamicin and tobramycin.

demonstrated (four patients with genotype 2, two patients with genotype 3, and two patients with genotype 7 ). Fifty percent of all patients acquiring colonisation with $P$ aeruginosa did so via cross-colonisation, the other $50 \%$ most probably being colonised from endogenous sources. In two of eight patients with VAP caused by $P$ aeruginosa, colonisation probably occurred via cross-colonisation. The three episodes of VAP within a short period of time that initiated this study were caused by three different genotypes of $P$ aeruginosa.

\section{COLONISATION PRESSURE AND ANTIBIOTIC}

PRESSURE

A tendency towards a higher rate of crosscolonisation in ICU 2 was observed - two of 39 patients (5\%) in ICU 1 and six of $54(11 \%)$ in ICU $2\left(p=0.2, \chi^{2}\right.$ test). The fact that more episodes of cross-colonisation occurred in ICU 2 may be related to the higher colonisation pressure in this ward. Mean (SD) colonisation pressure was $40(11) \%$ in ICU 2 and $35(9) \%$ in ICU 1 ( $p<0.05$, Mann-Whitney test). The mean antibiotic pressure for patients was comparable in both ICUs. However, mean antibiotic pressure was higher for patients who acquired colonisation than for those who did not $(68$ (45)\% and 37 (43)\%, respectively; $\mathrm{p}<0.05$; Mann-Whitney test).

\section{Discussion}

Our findings suggest that cross-colonisation may be an important route of colonisation and infection for $P$ aeruginosa even when overall 
incidence rates do not suggest the occurrence of an outbreak. The epidemiology of $P$ aeruginosa may therefore be described as a silent epidemic, sharing similarities with the epidemiology of other nosocomial pathogens such as vancomycin resistant enterococci, methicillin resistant Staphylococcus aureus, and ceftazidime resistant Gram negative bacilli. The results also show that surveillance in combination with molecular biotyping may be used as a method to perform continuous quality improvement. Finally, our findings emphasise the constant need for improving compliance with infection control measures, and warrant further studies to elucidate the relative importance of endogenous and exogenous colonisation with $P$ aeruginosa and to determine variables that influence both routes of colonisation and infection.

The incidence of VAP due to $P$ aeruginosa ( $12 \%$ of mechanically ventilated patients) during the period of the study is representative for our ICU. During 1994, the year following this study, 58 of $276(21 \%)$ mechanically ventilated patients admitted to our ICU developed VAP, in $28(48 \%)$ of the cases due to $P$ aeruginosa, either alone or in combination with other microorganisms - that is, $10 \%$ of all mechanically ventilated patients. ${ }^{15}$ In a large study performed in 1992 and 1993 in our ICU the incidence of VAP was $22 \%$, with $P$ aeruginosa involved in $58 \%$ of patients $(13 \%$ of all mechanically ventilated patients) ${ }^{16}$ The incidence of VAP has therefore remained fairly stable throughout the years. Moreover, the clinical observation of three episodes of VAP within a short period of time appeared to be caused by three different genotypes. The period under study therefore reflects our endemic setting and is not an outbreak. Comparison of incidence rates with those in other ICUs is difficult, mainly because of different patient populations. However, the incidence of VAP ranges from $15 \%$ to $24 \%$ in settings where VAP was also diagnosed by bronchoscopy, ${ }^{17-20}$ and the proportion caused by $P$ aeruginosa varied from $14 \%$ to $55 \%$. $^{17-22}$

Observations of similar genotypes of $P$ aeruginosa colonising and infecting several patients strongly suggest the occurrence of crosscolonisation, not only between patients in the same ward but also between patients from different wards. Although contaminated equipment might have been a source, regular examinations of nebulisers and sinks were always negative for $P$ aeruginosa, and the polyclonal character of the epidemiology make this possibility fairly unlikely.

The possibility of cross-colonisation of $P$ aeruginosa in non-epidemic situations has rarely been studied. Olson and coworkers ${ }^{23}$ addressed this issue in a study of 270 patients admitted to a medical/surgical ICU ward; 63 (23\%) patients were colonised with $P$ aeruginosa on admission and $33(16 \%)$ acquired colonisation, 12 of whom (36\%) were found to be due to cross-colonisation. In a prospective surveillance study carried out in five ICUs during a six month period, Chetchotisakd et $a l^{24}$ did not find an important role for cross-colonisation, only $14(10 \%)$ of 137 isolates of bacteria ( $P$ aeruginosa, $E$ coli, Klebsiella pneumoniae, Enterobacter cloacae, enterococci) cultured from patients with suspected infection being acquired by cross-colonisation. Although molecular biotyping methods were used to determine the identity of the isolates, they may have underestimated the true incidence of cross-colonisation. Only a single isolate from a suspected site of infection was analysed, without including isolates from colonised patients. It is evident that infections with $P$ aeruginosa only form the tip of the iceberg of the complete epidemiology, ${ }^{23}$ and its epidemiology can only be studied reliably when surveillance of colonisation is included. Moreover, patients may be colonised or infected with multiple genotypes of $P$ aeruginosa, and analysis of a single isolate will not accurately represent the whole epidemiology. ${ }^{25}$

The difference in the number of cases of cross-colonisation between both ICUs in the present study (two in ICU 1 and six in ICU 2) may be associated with the higher colonisation pressure in ICU 2 compared with ICU 1. However, differences in compliance with infection control measures, which was not monitored, may be another explanation for this difference. In addition, antibiotic pressure was clearly higher for patients who acquired $P$ aeruginosa. These findings suggest that both endogenous colonisation-for example, intestinal colonisation - and exogenous colonisation play a role in the epidemiology of respiratory tract colonisation with $P$ aeruginosa. In this regard, the epidemiology of $P$ aeruginosa may share similarities with endemic situations of other nosocomial pathogens such as vancomycin-resistant enterococci. These bacteria are also frequently acquired via cross-colonisation $^{26}$ and their acquisition seems to be influenced by a high colonisation pressure and a high antibiotic pressure, providing these bacteria with a selective growth advantage. ${ }^{27}$ However, the present study does not allow us to determine the precise relative impact of both exogenous and endogenous colonisation of $P$ aeruginosa. Since the study was started abruptly after the clinical suspicion of crosscolonisation, surveillance was not uniform in all patients. More importantly, no surveillance cultures of the rectum were performed. Since rectal colonisation may be a source of respiratory tract colonisation, ${ }^{72} 29$ cases of endogenous colonisation may have been missed. The relative importance of endogenous and exogenous colonisation in the pathogenesis of VAP therefore remains to be established.

The authors thank Monique Coomans and Christel Driessen for the microbiological analysis, and the medical and nursing staff of the ICU who helped to make this study possible. This study was supported in part by grant 28-2125-1 from the Praevention Foundation.

1 Fagon JY, Chastre J, Hance AJ, et al. Nosocomial pneumonia in ventilated patients: a cohort study evaluating attributable mortality and hospital stay. Am f Med 1993;94:2818 .

2 Bonten MJM, Bergmans DCJJ, Ambergen AW, et al. Risk factors for pneumonia, and colonization of respiratory tract Respir Crit Care Med 1996;154:1339-46. 
3 Bonten MJM, Gaillard CA, de Leeuw PW, et al. Role of colonization of the upper intestinal tract in the pathogenesis of ventilator-

4 Niederman MS, Craven DE. Editorial response: devising strategies for preventing nosocomial pneumonia. Shoul we ignore the stomach? Clin Infect Dis 1997;24:320-3.

5 Atherton ST, White DJ. Stomach as source of bacteria colonising respiratory tract during artificial ventilation. Lancet 1978;ii:968-9.

6 Torres A, El-Ebiary M, González J, et al. Gastric and pharyngeal flora in nosocomial pneumonia acquired during

7 Murthy SK, Baltch AI. Am Rev Respir Dis 1993;148:352-7. fecal carriage of Pseudomonas aeruginosa in hospital patients. F Clin Microbiol 1989;27:35-40.

8 Whitby JL, Rampling A. Pseudomonas aeruginosa contamination in domestic and hospital environment. Lancet tion in don $1972 ; \mathrm{i}: 15-7$.

9 Favero MS, Carson LA, Bond WW, et al. Pseudomonas aeruginosa: growth in distilled water from hospitals. Science aeruginosa: growth

10 Grundmann H, Kropec A, Hartung D, et al. Pseudomonas aeruginosa in a neonatal intensive care unit: reservoirs and ecology of the nosocomial pathogen. F Infect Dis 1993;168: 943-7.

11 Knaus WA, Draper EA, Wagner DP, et al. APACHE II: a severity of disease classification system. Crit Care Med 1985;13:818-29.

12 Bonten MJM, Gaillard CA, van der Hulst R, et al. Intermittent enteral feeding: the influence on respiratory and digestive tract colonization in mechanically ventilated intensivecare-unit patients. Am $\mathcal{F}$ Respir Crit Care Med 1996;154: 394-9.

13 Grothues D, Koopmann U, Hardt H, et al. Genome fingerprinting of Pseudomonas aeruginosa indicates colonization of cystic fibrosis siblings with closely related strains. $7 \mathrm{Clin}$ Microbiol 1988;26:1973-7.

14 Tenover FC, Arbeit RD, Goering RV, et al. Interpreting chromosomal DNA restriction patterns produced by chromosomal DNA restriction patterns produced by pulsed-field gel electrophoresis: criteria

15 Bergmans DCJJ, Bonten MJM, Gaillard CA, et al. Indications for antibiotic use in ICU patients: a one-year prospective surveillance. F Antimicrob Chemother 1997;39: 527-35.

16 Bonten MJM, Gaillard CA, van der Geest S, et al. The role of intragastric acidity and stress ulcer prophylaxis on colonization and infection in mechanically ventilated patients. A stratified, randomized, double blind study of sucralfate versus antacids. Am ₹ Respir Crit Care Med 1995;152:182534.
17 Fagon JY, Chastre J, Domart Y, et al. Nosocomial pneumonia in patients receiving continuous mechanical ventilation: prospective analysis of 52 episodes with use of a protected specimen brush and quantitative culture technique. $\mathrm{Am}$ Rev Respir Dis 1989;139:877-84.

18 Torres A, Aznar R, Gatell JM, et al. Incidence, risk, and prognosis factors of nosocomial pneumonia in mechanically ventilated patients. Am Rev Respir Dis 1990;142:5238.

19 Chevret S, Hemmer M, Carlet J, et al and the European Cooperative Group on Nosocomial Pneumonia. Incidence and risk factors of pneumonia acquired in intensive care units: results from a multicenter prospective study on 996 patients. Intens Care Med 1993;19:256-64.

20 Papazian L, Bregeon F, Thirion X, et al. Effect of ventilatorassociated pneumonia on mortality and morbidity. Am $\mathcal{F}$ Respir Crit Care Med 1996;154:91-7.

21 Craven DE, Steger KA, Barat LM, et al. Nosocomial pneumonia: epidemiology and infection control. Intens Care Med 1992;18:S3-9.

22 Bonten MJM, Gaillard CA, van Tiel FH, et al. The stomach is not a source for colonization of the upper respiratory ract and pneumonia in ICU patients. Chest 1994;105:87884.

23 Olson B, Weinstein RA, Nathan C, et al. Epidemiology of endemic Pseudomonas aeruginosa: why infection control efforts have failed. F Infect Dis 1984;150:808-16.

24 Chetchotisakd P, Phelps CL, Hartstein AI. Assessment of bacterial cross-transmission as a cause of infections in patients in intensive care units. Clin Infect Dis 1994;18:92937.

25 Bergmans D, Bonten M, van Tiel F, et al. Value of phenotyping methods as an initial screening of Pseudomonas aeruginosa in epidemiologic studies. Infection domonas aerugin:

26 Bonten M, Hayden MK, Nathan C, et al. Epidemiology of colonisation of patients and environment with vancomycinresistant enterococci. Lancet 1996;348:1615-9.

27 Bonten MJM, Slaughter S, Ambergen AW, et al. The role of 'colonization pressure' in the spread of vancomycinresistant enterococci: an important infection control variable. Arch Intern Med 1998;158:1127-32.

28 Noone MR, Pitt TL, Bedder M, et al. Pseudomonas aeruginosa colonisation in an intensive therapy unit: role of cross infection and host factors. BMF 1983;286:341-4.

29 Kropec A, Huebner J, Riffel M, et al. Exogenous or endogenous reservoirs of nosocomial Pseudomonas aeruginosa and Staphylococcus aureus infections in a surgical intensive care unit. Intens Care Med 1993;19:161-5. 